\section{Clarification regarding the statement of the association between the recombinant zoster vaccine (RZV) and gout flares}

We have read with great interest the article addressing the risk of gout flares after vaccination in a case crossover study by Yokose et al. ${ }^{1}$ The authors suggest that vaccines may be associated with an increased odds of gout flares potentially via activation of the NLRP3 inflammasome. The study was conducted between 2003 and 2010 prior to the availability of the recombinant zoster vaccine (RZV); however, the authors make reference to two phase III clinical trials for RZV which, they state, demonstrated a higher risk of gout flares in the vaccine group. Even though RZV was not assessed in the study by Yokose et al, we would like to clarify this statement about RZV and gout flares.

The two large pivotal phase III clinical trials, ZOSTER-006/ ZOE-50 (NCT01165177) and -022/ZOE-70 (NCT01165229), that involved a total of 29305 subjects $\geq 50$ years of age who received at least one dose of RZV $(n=14645)$ or placebo $(n=14660)$ were designed to assess the efficacy and safety of the RZV vaccine, but not to statistically assess a potential risk of gout among RZV recipients. ${ }^{2-4}$

We confirm that the analysis of the unsolicited adverse events (AE) reported during 30 days after each vaccination showed a numerical imbalance in the reporting rate of gout. Indeed, there were $27(0.18 \%$ (95\% CI 0.12 to 0.27$))$ versus $8(0.05 \%$ (95\% CI 0.02 to 0.11$)$ ) (unadjusted risk rario $=3.38(95 \%$ CI 1.49 to 8.60)) subjects in the RZV and placebo groups, respectively, who experienced an $\mathrm{AE}$ of gout or gouty arthritis. ${ }^{5}$ However, these are not necessarily reported 'flares' as it includes both newly diagnosed (or incident) gouts and recurrent gouts (potential flares). Any AE was collected regardless of whether it was newly diagnosed or recurrent. As part of the safety monitoring, the risk of a newly diagnosed gout was analysed separately from the risk of a recurrent gout in a descriptive analysis. Differentiation between incident and recurrent gouts was done by retrospective review of the documented patient's medical history. It is worth noting that GSK may not have had access to full patient medical history information, including that classically used for diagnosis ascertainment, such as baseline serum uric acid levels or presence of monosodium urate crystals in synovial fluid.

It is important to note that the total number of cases of incident and recurrent gout reported was low (35 reported cases in the pooled analysis). Majority of the gout events in the RZV group were non-serious and mild-to-moderate in severity. Of the total of patients reporting an episode of gout after vaccination, 19 in the RZV group and 3 in the placebo group reported an episode of gout for the first time, while 8 subjects in the RZV group versus 5 subjects in the placebo group were reportedly known to have pre-existing (chronic) gout at baseline and experienced a recurrent episode of gout (gout flare) after vaccination. ${ }^{6}$ From the data, it appears that newly diagnosed episodes of gout were more frequent than acute gout flares, regardless of gout stage (ie, intercritical period or currently having gouty arthritis or chronic tophaceous gout). However, available data cannot be used to draw conclusions in this regard, since majority were non-serious reports for which there is inconsistent clinical data completeness for assessment, for example, in terms of dietary habits, baseline serum uric acid levels, medical history, clinical narrative and diagnostic tests. In addition, multiple factors contribute to pathophysiology of gout and the individuals who reported episodes of gout after vaccination also had various confounding factors and medical conditions that are well-known risk factors for gout (eg, diabetes, hypertension, chronic kidney disease, hypercholesterolaemia, therapy with digoxine, $\beta$-blockers, diuretics, etc), and which may have explained the occurrence of the reported gout attack. Given that the two large pivotal phase III clinical trials were not designed to statistically assess a potential risk of gout among RZV recipients, one cannot exclude that the observed imbalance might be a chance finding, considering that the prevalence of gout in RZV target population (adults 50 years of age or older) is common. ${ }^{5}$

In view of all these caveats, the numerical imbalance observed should be interpreted with caution, and further data are needed to investigate a possible putative association between RZV vaccination and increased risk of gout, if any. The RZV postmarketing safety surveillance includes currently under development targeted safety studies designed to investigate the risk of incident gout.

Regarding the hypothesis of a potential mechanistic link between gout and NLRP3 inflammasome activation, it should be noted that, although activation of this pathway has been reported in vitro, it is controversial whether it has a role in the adjuvant effect of alum in vivo. ${ }^{7}$ Similarly, QS-21 is able to trigger activation of NLRP3 in vitro and caspase- 1 cleavage in the lymph node draining the injection site, ${ }^{8}$ but studies in NLRP3-deficient mice showed that this pathway has no impact on the adjuvant effect of QS-21 or QS-21-containing adjuvants in vivo. ${ }^{9} 10$ It is important to note that, for the vaccine to be the trigger of gout symptoms, it would have to directly affect the joint environment where the inflammation occurs. Given that the immune-stimulatory effect is local to the site of injection, ${ }^{11}$ a direct trigger of gout by adjuvanted vaccines through caspase- 1 activation is an unlikely hypothesis. Uric acid may be produced locally as a result of vaccine-induced inflammatory response through the release of DNA by dying innate cells (such as neutrophils) after they have been recruited at the site of injection. This has been shown to play a role in the adjuvant effect of alum. ${ }^{12}$ It is not known, however, whether this effect occurs in humans and whether it would be significant enough to cause an increase in uric acid level in blood. Therefore, assessing changes in circulating uric acid blood level postvaccination could help verify this hypothesis, keeping in mind that an increase in serum uric acid levels (asymptomatic hyperuricaemia) may not necessarily translate into monosodium urate crystal formation in the joints and acute gout.

While the article by Yokose et $a l^{1}$ proposed a potential association between vaccines and gout flares, the authors also highlighted methodological and statistical limitations of the study (eg, self-reporting, representativeness of the population, lack of information on the type of vaccine administered) that should be considered when drawing conclusions based on the Yokose results. A prospective study designed to measure multiple variables, such as age and sex, medical history of gout and medications, exact time of immunizations, vaccine used, reactogenicity and so on would help assess possible risks of gout in the context of vaccination. Any finding of an association should then be complemented with mode of action and mechanistic studies designed to understand potential putative mechanisms.

Finally, we concur with the authors on the importance of what vaccination brings to public health and the continuous need to evaluate the benefit/risk balance of vaccines.

Arnaud M Didierlaurent $\odot,{ }^{1}$ Christophe Desssart, ${ }^{1}$
Anthony L Cunningham ${ }^{2}$
${ }^{1}$ GSK, Wavre, Belgium
${ }^{2}$ Centre for Virus Research, The Westmead Institute for Medical Research, University
of Sydney, Australia, Westmead, New South Wales, Australia 
Correspondence to Dr. Arnaud M Didierlaurent, GSK, Wavre 1300, Belgium; arnaud.x.didierlaurent@gsk.com

Acknowledgements The authors thank Fernanda Tavares da Silva, Carla Talarico, Robbert Van Der Most, Anne Schuind, Robyn Widenmaier and Martina Kovac Choma (GSK) for the critical review of this letter. Editorial assistance and publication coordination were provided by Natalia Tumanova (Modis; Wavre, Belgium; on behalf of GSK).

Contributors All authors contributed to the conception of the draft, draft preparation and revision, approving the final version for submission.

Funding GlaxoSmithKline Biologicals SA covered the costs associated with the development and publishing of this letter.

Competing interests $A M D$ and CD are GSK employees and own shares in the GSK group of companies. ALC reports funding to his institution from Merck, BioCSL/ Sequirus and GSK outside the submitted work.

Patient consent for publication Not required.

Provenance and peer review Not commissioned; internally peer reviewed.

(c) Author(s) (or their employer(s)) 2019. No commercial re-use. See rights and permissions. Published by BMJ.

\section{(A) Check for updates}

To cite Didierlaurent AM, Desssart C, Cunningham AL. Ann Rheum Dis Epub ahead of print: [please include Day Month Year]. doi:10.1136/annrheumdis-2019-216639

Received 13 November 2019

Accepted 17 November 2019

Ann Rheum Dis 2019;0:1-2. doi:10.1136/annrheumdis-2019-216639

ORCID iD

Arnaud M Didierlaurent http://orcid.org/0000-0002-4736-9003

\section{REFERENCES}

1 Yokose C, McCormick N, Chen C, et al. Risk of gout flares after vaccination: a prospective case cross-over study. Ann Rheum Dis 2019;78:1601-4.

2 Lal H, Cunningham AL, Godeaux O, et al. Efficacy of an adjuvanted herpes zoster subunit vaccine in older adults. N Eng/ J Med 2015;372:2087-96.

3 Cunningham AL, Lal H, Kovac M, et al. Efficacy of the herpes zoster subunit vaccine in adults 70 years of age or older. N Engl J Med 2016;375:1019-32.

4 López-Fauqued M, Campora L, Delannois F, et al. Safety profile of the adjuvanted recombinant zoster vaccine: pooled analysis of two large randomised phase 3 trials. Vaccine 2019;37:2482-93.

5 Glaxosmithkline biologicals. Briefing document: Shingrix (zoster vaccine recombinant, adjuvanted) vaccines and related biological products Advisory Committee, 2017. Available: https://www.fda.gov/media/107553/download

6 Bla clinical review memorandum, 2017. Available: https://www.fda.gov/media/ 108793/download

7 Spreafico R, Ricciardi-Castagnoli P, Mortellaro A. The controversial relationship between NLRP3, alum, danger signals and the next-generation adjuvants. Eur $\int$ Immunol 2010;40:638-42.

8 Detienne S, Welsby I, Collignon C, et al. Central Role of CD169' Lymph Node Resident Macrophages in the Adjuvanticity of the QS-21 Component of AS01. Sci Rep 2016;6:39475

9 Marty-Roix R, Vladimer GI, Pouliot K, et al. Identification of QS-21 as an Inflammasome-activating molecular component of saponin adjuvants. J Biol Chem 2016:291:1123-36.

10 Wilson NS, Duewell P, Yang B, et al. Inflammasome-Dependent and -independent IL-18 production mediates immunity to the ISCOMATRIX adjuvant. J Immunol 2014;192:3259-68.

11 Didierlaurent AM, Collignon C, Bourguignon P, et al. Enhancement of adaptive immunity by the human vaccine adjuvant AS01 depends on activated dendritic cells. $J$ Immunol 2014;193:1920-30.

12 Kool M, Soullié T, van Nimwegen M, et al. Alum adjuvant boosts adaptive immunity by inducing uric acid and activating inflammatory dendritic cells. J Exp Med 2008:205:869-82. 